\section{Research Square}

\title{
Comparison of nutritional effectiveness and complication rate between early nasojejunal and nasogastric tube feeding in patients with an intracerebral hemorrhage
}

\section{Cong Ling}

Third Affiliated Hospital of Sun Yat-Sen University

Xiling Hu

Third Affiliated Hospital of Sun Yat-Sen University

\section{Chuan Chen}

Third Affiliated Hospital of Sun Yat-Sen University

\section{Lun Luo}

Third Affiliated Hospital of Sun Yat-Sen University

\section{Meiqin Cai}

Third Affiliated Hospital of Sun Yat-Sen University

\section{Chaofeng Liang}

Third Affiliated Hospital of Sun Yat-Sen University

\section{Hui Wang}

Third Affiliated Hospital of Sun Yat-Sen University

Ying Guo (D Doctor200@163.com )

Third Affiliated Hospital of Sun Yat-Sen University

\section{Research}

Keywords: Enteral nutrition, intracerebral hemorrhage, nasojejunal feeding tube, nasogastric feeding tube

Posted Date: May 13th, 2020

DOI: https://doi.org/10.21203/rs.3.rs-27456/v1

License: (c) (i) This work is licensed under a Creative Commons Attribution 4.0 International License. Read Full License 


\section{Abstract}

\section{Background}

This study aimed to compare nutritional effectiveness and complication rate between early nasojejunal and nasogastric tube feeding in patients with an intracerebral hemorrhage.

\section{Methods}

Eighty patients with an intracerebral hemorrhage were randomly divided into a nasojejunal and a nasogastric tube feeding group. Feeding tubes were placed within 6 hours after admission, and enteral feeding began within 2 hours after tube placement. The nutritional status and complication rate of the 2 groups were compared before and 2 and 4 weeks after beginning feeding.

\section{Results}

Serum prealbumin, serum albumin, and hemoglobin levels were significantly higher in the nasojejunal tube feeding group than in the nasogastric tube feeding group at 2 and 4 weeks after beginning feeding (all, $p<0.05)$. The incidence of gastric retention, pulmonary aspiration, and pneumonia were lower in the early nasojejunal tube feeding group than in the early nasogastric tube feeding group (all, $p<0.05$ ). There was no significant difference in the incidence of diarrhea between the 2 groups.

\section{Conclusion}

Compared with early nasogastric feeding, early nasojejunal feeding provides better nutritional effectiveness and a lower incidence of gastric retention, pulmonary aspiration, and pneumonia in patients with an intracerebral hemorrhage.

\#These authors contributed equally to this work.

\section{Background}

Patients with intracerebral hemorrhage have increased nutritional requirements, elevated protein catabolism, and compromised immunity. These patients also present with disorders of consciousness, facial paralysis, and swallowing disturbance, which are all associated with an increased incidence of pneumonia. Furthermore, $50 \%$ of patients with an intracerebral hemorrhage also have respiratory infections [1, 2].

Early and appropriate enteral nutrition provides adequate energy for patients to respond to a variety of stressors, maintains the body's immune function, and prevents changes in intestinal flora, and as such it can greatly reduce the incidence of infectious complications [3]. While early enteral nutrition is an important measure to prevent hospital-acquired pneumonia and reduce mortality and morbidity in patients with intracerebral hemorrhage, critically ill adultsonly receive $49-70 \%$ of their nutritional requirements [4]. Gastrointestinal feeding intolerance is a major obstacle for achieving adequate nutrition. Furthermore, an improper enteral nutrition route will significantly increase the incidence of gastrointestinal feeding intolerance.

Nasogastric tube feeding is the most commonly used route for providing enteral nutrition. However, the incidence of gastric retention is high and is responsible for $13-44 \%$ of pneumonia cases [5-7]. In theory, a nasojejunal tube feeding reduces gastroesophageal reflux, maintains gastrointestinal function, ensures an adequate intake of nutrients, and reduces the risk of aspiration pneumonia [8]. In the past, placement of jejunal feeding tubes often required X-ray or endoscopic guidance. Factors such as patient transportation, long operative times, and high medical costs can delay the initiation of enteral nutrition and increase the risk of complications [9]. Thus, these factors can compromise the benefits of nutritional support. 
Studies, however, have reported no difference in the incidence of pneumonia between patients receiving jejunal tube feeding and gastric tube feeding [10-12].

Thus, the purpose of this study was to compare the nutritional effectiveness and incidence of complications of early nasojejunal tube feeding and early nasogastric tube feeding in patients with an intracerebral hemorrhage.

\section{Methods \\ Patients}

This study was reviewed and approved by the ethics committee of our medical center, and written consent was obtained from family members or guardians of each patient.

From August 2015 to August 2016, patients with an intracerebral hemorrhage admitted and treated at our center, and meeting the inclusion criteria, were included in the study. Patients included in the study were randomized into 2 feeding groups: early nasojejunal tube feeding and early nasogastric tube feeding.

\section{Inclusion and Exclusion Criteria}

Inclusion criteria were over 18 years of age diagnosed with intracranial hemorrhage by a computed tomography (CT) scan, and a Glasgow Coma Scale (GCS) score < 13 points. Only patients observed for more than 2 weeks were included in the study.

Exclusion criteria were: 1) History of respiratory or gastrointestinal diseases; 2) An endocrine disease that impacts nutritional metabolism; 3) Received a craniotomy during hospitalization; 4) Required mechanical ventilation before a diagnosis of pneumonia was made; 5) Died within 2 weeks after admission. Rationale for the exclusion criteria included: 1) Ahistory of a respiratory disease is an independent predictor of pneumonia. 2) Gastrointestinal diseases or metabolic endocrine disorders can affect nutritional status. 3) Surgery and mechanical ventilation can cause pneumonia.

\section{Feeding Tube Placement Procedure}

All procedures were performed at the bedside by senior clinicians with 7 or more years of experience. In the nasogastric feeding group, the patient was placed in a semi-recumbent position, the gastric tube was slowly inserted through the nostril to a length of $55-60 \mathrm{~cm}$. Then a syringe was used to withdraw the gastric juice to confirm that the gastric tube was properly placed in the stomach.

In the jejunal feeding group, the nasojejunal feeding tube was placed within 6 hours after admission. Patients were placed in a semi-recumbent position with $30-45^{\circ}$ elevation of the head of the bed. Ondansetron, $8 \mathrm{mg}$, was administered by intravenous injection 30 minutes before the procedure. The procedure used to place gastric tube in the nasogastric feeding group were used to place the nasojejunal feeding tube in the stomach. Then, $10 \mathrm{ml}$ of normal saline was injected into the balloon of the jejunal feeding tube, which slowly entered the jejunum with an insertion depth of about $110 \mathrm{~cm}$ via the force of gastric peristalsis. The tube was then appropriately fixed to one side of the nose with adhesive tape.

In both groups, a radiograph was taken after tube placement to confirm the position of the tube tip.

\section{Nutritional Regimen}


The nutritional regimen was the same in both groups. Enteral nutrition was initiated within 2 hours after placement of the feeding tube. The calorie target was $25 \mathrm{kcal} / \mathrm{kg} / \mathrm{day}$, and the nitrogen uptake target was $0.2 \mathrm{~g}$ nitrogen $/ \mathrm{kg} / \mathrm{day}$ [13]. In both groups, $50 \%$ of the target calories were administered on the first day, $80 \%$ on the second via gastric or nasojejunal tubes, and $100 \%$ on the third day and thereafter through the parenteral route. The nutrients $\left(37-40{ }^{\circ} \mathrm{C}\right)$ were pumped into the feeding tube by an enteral feeding system (Baitong, Qingdao Ruicong Medical Equipment Co., Ltd., China) at an even speed of $60-80 \mathrm{~mL} /$ hour. Gastrointestinal fluid was withdrawn every 6 hours, and tested with pH paper to determine whether the feeding tube was in the correct position. $\mathrm{A} \mathrm{pH}$ value of $<6$ indicated the tip of the feeding tube was in the stomach [14]. A $\mathrm{pH}$ value of $>7$ indicated the tip of the feeding tube was in the duodenum/jejunum [9]. If gastrointestinal retention or vomiting occurred, the enteral feeding was paused for 6 hours.

\section{Evaluation of Nutritional Status and Complications}

Serum prealbumin, serum albumin, and hemoglobin were used as indicators of nutritional status. They were measured 1 day prior to beginning enteral feeding, and 2 and 4 weeks after beginning enteral feeding.

Complications considered in this study were gastric retention, pulmonary aspiration, diarrhea, and pneumonia. The diagnostic criteria for the complications were the same as used in a prior study [13]. Gastric retention was defined as > $200 \mathrm{ml}$ of fluid withdrawn from the feeding tube. Pulmonary aspiration was defined as sudden choking, difficulty breathing, or coughing up nutrient solution-like sputum. Diarrhea was defined as the presence of loose or watery stool, frequent and loud bowel sounds, and 3 or more bowel movements per day.

If pneumonia was suspected bronchial alveolar lavage was performed to obtain lavage fluid for pathogen culture. A diagnosis of pneumonia required a new and persistent infiltrate on chest radiograph, and at least 2 of the following: 1) A body temperature $>38.3^{\circ} \mathrm{C} ; 2$ ) Leukocytosis, defined as a white blood cell (WBC) count increased by $25 \%$ or more from baseline and a value $>10,000 \mathrm{WBC} / \mathrm{mm}^{3}$, or leucopenia, defined as a WBC count decreased by more than $25 \%$ from baseline and $<5,000 \mathrm{WBC} / \mathrm{mm}^{3}$; 3) Gram stain of a purulent tracheal aspirate showing $>25 \mathrm{WBC}$ per high-power field. A positive bronchoalveolar lavage fluid culture was not required for a diagnosis of pneumonia, but was considered to support a pneumonia diagnosis.

\section{Statistical analysis}

Quantitative data with a normal distribution were expressed as mean \pm standard error of the mean (SEM). Differences between groups were compared with the t-test. Complication rates were compared with the $\chi^{2}$ test. A value of $p<0.05$ was considered to indicate statistical significance. Statistical analyses were performed with SPSS version 17.0 statistical software.

\section{Results}

From August 2015 to August 2016, 106 patients with an intracerebral hemorrhage treated at our center, and 26 cases were excluded based on the exclusion criteria. Finally, 80 eligible patients were included in the study, and there were 37 in the early nasojejunal group and 43 in the early nasogastric group. Patient age, sex, Acute Physiologic Assessment and Chronic Health Evaluation (APACHE) II score, GCS score, and hemorrhage volume were similar between the 2 groups (all, $p>0.05$ ) (Table 1). 
Table 1

Characteristics of the early nasojejunal and early nasogastric groups

\begin{tabular}{|llll|}
\hline & Early nasojejunal group & Early nasogastric group & p-value \\
\hline Number of patients & 40 & 40 & \\
\hline Age, years & $55.4 \pm 16.1$ & $53.8 \pm 15.4$ & 0.665 \\
\hline Females & $8(20 \%)$ & $10(25 \%)$ & 0.287 \\
\hline APACHE II score & $14.4 \pm 1.8$ & $14.3 \pm 1.6$ & 0.765 \\
\hline GCS score & $8.5 \pm 1.1$ & $8.7 \pm 1.2$ & 0.464 \\
\hline Bleeding volume $(m l)$ & $31 \pm 12$ & $34 \pm 14$ & 0.334 \\
\hline Data presented as mean \pm standard error of the mean, or number (percentage). & \\
\hline APACHE, Acute Physiologic Assessment and Chronic Health Evaluation; GCS, Glasgow Coma Scale. \\
\hline
\end{tabular}

Serum prealbumin, serum albumin, and hemoglobin levels before tube feeding were similar between the early nasojejunal and early nasogastric feeding groups (all, $p>0.05$ ). However, serum prealbumin, serum albumin, and hemoglobin levels were significantly higher in the nasojejunal feeding group than in the nasogastric feeding group at 2 and at 4 weeks after beginning enteral feeding (all, $\mathrm{p}<0.05)$ (Table 2$)$.

Table 2

Laboratory assessments at different time points

\begin{tabular}{|c|c|c|c|c|c|c|c|c|c|}
\hline & \multicolumn{3}{|c|}{ Before feeding } & \multicolumn{3}{|c|}{$\begin{array}{l}2 \text { Weeks after beginning } \\
\text { feeding }\end{array}$} & \multicolumn{3}{|c|}{4 Weeks after beginning feeding } \\
\hline & $\begin{array}{l}\text { Serum } \\
\text { prealbumin }\end{array}$ & $\begin{array}{l}\text { Serum } \\
\text { albumin }\end{array}$ & $\mathrm{Hgb}$ & $\begin{array}{l}\text { Serum } \\
\text { prealbumin }\end{array}$ & $\begin{array}{l}\text { Serum } \\
\text { albumin }\end{array}$ & $\mathrm{Hgb}$ & $\begin{array}{l}\text { Serum } \\
\text { prealbumin }\end{array}$ & $\begin{array}{l}\text { Serum } \\
\text { albumin }\end{array}$ & $\mathrm{Hgb}$ \\
\hline $\begin{array}{l}\text { Early } \\
\text { nasojejunal } \\
\text { group* }\end{array}$ & $0.32 \pm 0.03$ & $\begin{array}{l}38.0 \pm \\
4.0\end{array}$ & $\begin{array}{l}143 \\
\pm 5.5\end{array}$ & $0.28 \pm 0.04$ & $\begin{array}{l}35.5 \pm \\
3.8\end{array}$ & $\begin{array}{l}135 \\
\pm 4.6\end{array}$ & $0.27 \pm 0.04$ & $\begin{array}{l}34.8 \pm \\
3.6\end{array}$ & $\begin{array}{l}128 \pm \\
4.8\end{array}$ \\
\hline $\begin{array}{l}\text { Early } \\
\text { nasogastric } \\
\text { group* }\end{array}$ & $0.31 \pm 0.04$ & $\begin{array}{l}37.5 \pm \\
4.3\end{array}$ & $\begin{array}{l}144 \\
\pm 5.2\end{array}$ & $0.25 \pm 0.05$ & $\begin{array}{l}34.0 \pm \\
3.7\end{array}$ & $\begin{array}{l}131 \\
\pm 5.3\end{array}$ & $0.23 \pm 0.05$ & $\begin{array}{l}33.6 \pm \\
3.4\end{array}$ & $\begin{array}{l}118 \pm \\
4.5\end{array}$ \\
\hline p-value & 0.402 & 0.992 & 0.136 & 0.001 & 0.012 & 0.001 & 0.003 & 0.009 & $\begin{array}{l}< \\
0.0010\end{array}$ \\
\hline \multicolumn{10}{|c|}{ Data presented as mean \pm standard error of the mean. } \\
\hline \multicolumn{10}{|c|}{ Serum prealbumin, g/L; Serum albumin, g/L; Hgb, g/L. } \\
\hline \multicolumn{10}{|c|}{ Hgb, hemoglobin. } \\
\hline$*_{n}=40$ in & roun & & & & & & & & \\
\hline
\end{tabular}

The incidence of gastric retention, pulmonary aspiration, and pneumonia were all lower in the early nasojejunal feeding group than in the early nasogastric feeding group (all, $p<0.05)$ (Table 3$)$. The incidence of diarrhea was not significantly different between the 2 groups $(p>0.05)$. 
Table 3

Comparison of complications in the 2 groups

\begin{tabular}{|lllll|}
\hline & Gastric retention & Pulmonary aspiration & Diarrhea & Pneumonia \\
\hline Early nasojejunal group* & $3(7.5 \%)$ & $2(5 \%)$ & $5(12.5 \%)$ & $2(5 \%)$ \\
\hline Early nasogastric group* & $14(35 \%)$ & $9(22.5 \%)$ & $4(10 \%)$ & $8(20 \%)$ \\
\hline p-value & 0.003 & 0.02 & 1.00 & 0.04 \\
\hline Data presented as number (percentage). & & & \\
\hline$*_{n}=40$ in each group. & & & \\
\hline
\end{tabular}

\section{Discussion}

Nutrition support has an important impact on the prognosis of critically ill patients, and enteral nutrition (EN) is increasingly recognized as an integral part of the management of critically ill patients. Early EN has been shown to reduce disease severity, maintain gastrointestinal (GI) physiology, and regulate the immune system [15].A meta-analysis shows that postpyloric feeding can increase daily caloric intake in critically ill patients as compared with gastric feeding. However, it is controversial whether it can reduce the incidence of pneumonia. There is no significant difference in mortality, ICU stay time and complication rate between the two feeding methods [15]. Most of the previous studies were carried out in the ICU, and patients were not screened for the type of diseases. It was difficult to compare the severity of the disease among patients with different diseases. At the same time, many studies have not ruled out the effects of other treatments on the above indicators, such as the depth of sedation [16], It may be more likely to determine the duration of mechanical ventilation and ICU hospital stay as compared with the development of pneumonia. In this study, patients with cerebral hemorrhage undergoing surgery and mechanical ventilation were excluded to minimize the impact of other factors on the results. There were no significant differences in age, gender, APACHEX score, GCS score, and bleeding volume between the two groups.

Studies have shown that most critically ill patients receiving gastrointestinal feeding receive only $50-70 \%$ of the target calorie and protein load [17-19]. However, a meta-analysis has shown that enteral feeding delivers better or similar calories per day as compared with gastric feeding [15]. It has been suggested that delivery of nutrition into the small bowel using a nasojejunal tube may be preferable to nasogastric feeding since the small bowel has better absorptive capacity 7 and motility,8 leading to fewer interruptions [20]. In this study, no significant differences in nutritional status parameters were noted between the 2 groups prior to beginning enteral feeding. However, while the values of all indices in both groups were lower at 2 weeks and 4 weeks after beginning feeding, the values in the nasojejunal tube feeding group were significantly greater than in the nasogastric tube feeding group at both 2 and 4 weeks. The results of this study demonstrated that nasojejunal feeding can provide more effective nutritional support than nasogastric feeding for patients with an intracerebral hemorrhage. Acutely ill patients lose an average of $5-10 \%$ of skeletal muscle mass per week during the ICU stay. Even among paralyzed patients, some trauma patients can increase energy consumption by $20-30 \%$. Weight loss, negative nitrogen balance, and immune dysfunction are characteristic responses of critically ill patients, which can easily induce acute inflammation and infectious complications, leading to increased incidence and mortality of pneumonia [21]. Rice and colleagues reported that patients who received fewer calories were less likely to return to independent living on discharge [22]. Therefore, we think that providing adequate nutritional support for critically ill patients is vitally important to protect the body's immune function and reduce the risk of pneumonia, and better nutritional support may be conducive to the rehabilitation of patients with an intracerebral hemorrhage. Of particular note is that our results suggest that patients with an intracerebral hemorrhage have substantial nutritional needs, and that our nutritional plan did not fully meet the needs of the patients. As such, patients with intracerebral hemorrhage should be provided greater nutrition than that provided in this study. 
Complications of enteral nutrition can compromise nutritional treatment and result in failure to reach nutritional goals. The reported incidence of gastric retention in patients with an intracerebral hemorrhage is $>80 \%$ [4]. Critically ill patients are frequently sedated, and sedation with morphine and midazolam has been show to slow gastric emptying and increase retention [23].In the event of gastric retention or pulmonary aspiration, the administration of enteral nutrition may be interrupted and thus patients will not meet calorie goals. Furthermore, if pneumonia occurs the nutrient requirement will increase greatly. Some studies have suggested that trauma and surgery can inhibit gastrointestinal emptying and peristalsis, causing esophageal-cardia sphincter relaxation [24, 25]. In these patients neither nasogastric feeding nor gastrostomy feeding can effectively reduce gastric retention and gastroesophageal reflux [26]. Under conditions of stress, the functions of the stomach and colon are mainly affected, whereas the functions of the jejunum and ileum are rarely affected [27]. Mentec et al. [25] demonstrated that patients with feeding intolerance due to delayed gastric emptying exhibit relatively normal small intestine function.

In the current study, the incidence of gastric retention and pulmonary aspiration were lower in the early nasojejunal group than in the nasogastric group, whereas the incidence of diarrhea was not significantly different between the 2 groups. We agree that intracerebral hemorrhage may cause varying degrees of cardiac esophageal sphincter relaxation, and inhibit gastrointestinal emptying and peristalsis. Thus, delayed gastric emptying may be present in patients with an intracerebral hemorrhage due to impaired brain function [13]. Delayed gastric emptying in patients receiving nasogastric tube feeding results in gastric retention, which may lead to reflux and pulmonary aspiration. With nasojejunal tube feeding, however, the food directly enters the jejunum without passing through the stomach and the relatively normal function of the small intestine can move food distally to reduce the incidence of reflux. In addition, the pylorus blocks food from entering the stomach in a retrograde manner, which reduces the potential for gastric retention. This study shows that the incidence of gastric retention was significantly lower in the jejunal feeding group than in the gastric feeding group, which can reduce the interruption of feeding, increase the supply of nutrients, and accelerate the achievement of nutrition goals.

Reflux and pulmonary aspiration are important causes of pneumonia. Pneumonia is a common complication in patients with an intracerebral hemorrhage, and can seriously affect the prognosis of these patients. An indwelling nasogastric tube increases the risk of pneumonia in patients with an intracerebral hemorrhage. In this situation, there are a number of mechanisms that contribute to the development of pneumonia. 1) Reflexive pharyngeal swallowing can normally prevent pulmonary aspiration; however, this mechanism can be dysfunctional in patients with an intracerebral hemorrhage and thus increase the risk of aspiration [28]. 2) Dysfunction of the upper and lower esophageal sphincters can be causes by the presence of a nasogastric tube, and thus increase the risk of gastroesophageal reflux and aspiration [29]. An indwelling nasogastric tube can cause gastric flora to migrate to the oropharynx and lower respiratory tract, a hypothesized gastropulmonary route of infection [30]. It has been suggested that the incidence of microaspiration can be reduced by placing the feeding tube below the stomach [31]. Similarly, this study also showed that the incidence of aspiration by jejunal feeding was reduced, which helped prevent pneumonia.

We found the incidence of pneumonia was significantly lower in the nasojejunal feeding group. We can postulate a number of reasons for this finding. 1) As mentioned above, the nutritional status of patients who received nasojejunal tube feeding was significantly better than those who received nasogastric tube feeding, and sufficient nutritional support can protect immune function. 2) Reflux and pulmonary aspiration were greater in patients receiving nasogastric tube feeding, and both are associated with pneumonia. 3) Reflux and pulmonary aspiration also led to the suspension or interruption of enteral feeding, and thus reduce the effectiveness of nutrition therapy. Inadequate nutritional support can weaken the immune system functions, and thus increase the risk pneumonia. Subsequently, pneumonia greatly increases the demand for nutrition and exacerbates nutritional deficiencies, thus forming a vicious cycle.

There are limitations to this study that should be considered. The number of patients was relatively small; however, the inclusion criteria were strict to eliminate the influence of confounding factors such as type of disease, and surgery and mechanical ventilation. While it is well-known that pulmonary aspiration can lead to pneumonia, we did not study the 
relations between the features or amount of pulmonary aspirate and the development of pneumonia. This study only compared the incidence and related factors of pneumonia but did not compare the ICU stay time, recovery degree and mortality rate between the two groups.

A previous study has shown that due to the high technical requirements, the success rate of nasojejunal tube placement in some hospitals is relatively low, delaying the start of early nutrition and the time to achieve nutrition goals [15].

Nevertheless, most studies did not mention the time and success rate of nasojejunal tube placement. This may also be one of the reasons for the contradiction between previous research results. Deane et al. believe that only those institutions capable of quickly placing nasojejunal jejunal feeding tube can generally improve nutrition intake [16]. In our study, the medical staffs who performed the jejunal tube placement were all well-trained and experienced, and the success rate of the tube placement was $100 \%$. But we did not calculate the time taken for the tube placement.

\section{Conclusion}

Compared with early nasogastric feeding, early nasojejunal feeding provides better nutritional effectiveness and a lower incidence of gastric retention, pulmonary aspiration, and pneumonia in patients with an intracerebral hemorrhage. The lower incidence of pneumonia may be associated with increased nutrient supply and reduced gastrointestinal intolerance. However, it is uncertain whether this conclusion can be applied to patients with other critical diseases. For medical units with inexperienced placement of jejunal tube, we do not recommend it as a routine method.

\section{Abbreviations}

CT: computed tomography; GCS:Glasgow Coma Scale; WBC:white blood cell; SEM:standard error of the mean; APACHE:Acute Physiologic Assessment and Chronic Health Evaluation; EN:enteral nutrition; GI gastrointestinal

\section{Declarations}

\section{Ethics approval and consent to participate}

This study was reviewed and approved by the ethics committee of the Third Affiliated Hospital of Sun Yat-sen University, and written consent was obtained from family members or guardians of each patient.

\section{Consent for publication}

A written consent was obtained from family members or guardians of each patient for publication.

\section{Availability of data and materials}

All the data and material were presented in the main paper.

\section{Competing interests}

The authors declare that they have no competing interests.

\section{Funding}

None declared. 


\section{Authors' contributions}

We declare that all the listed authors have participated actively in the study and all meet the requirements of the authorship. YG designed the study and wrote the protocol. CL, XLH and CC acquired the date. LL, MQC, CFL and HW analyzed and interpreted the data. CL was a major contributor in writing the manuscript. All authors read and approved the final manuscript.

\section{Acknowledgements}

None declared.

\section{References}

1. Dempsey DT, Mullen JL, Buzby GP. The link between nutritional status and clinical outcome: Can nutritional intervention modify it? Am. J. Clin. Nutr. 1988. p. 352-6.

2. Park S, Yang N, Seo E. The Effectiveness of Lumbar Cerebrospinal Fluid Drainage to Reduce the Cerebral Vasospasm after Surgical Clipping for Aneurysmal Subarachnoid Hemorrhage. J Korean Neurosurg Soc. 2015;57:167-73.

3. Elhassan AO, Tran LB, Clarke RC, Singh S, Kaye AD. Total Parenteral and Enteral Nutrition in the ICU: Evolving Concepts. Anesthesiol. Clin. 2017. p. 181-90.

4. Cahill NE, Dhaliwal R, Day AG, Jiang X, Heyland DK. Nutrition therapy in the critical care setting: What is "best achievable" practice? An international multicenter observational study. Crit Care Med. 2010;38:395-401.

5. Mann G, Hankey GJ, Cameron D. Swallowing function after stroke: Prognosis and prognostic factors at 6 months. Stroke. 1999;30:744-8.

6. Hilker R, Poetter C, Findeisen N, Sobesky J, Jacobs A, Neveling M, et al. Nosocomial pneumonia after acute stroke: Implications for neurological intensive care medicine. Stroke. 2003;34:975-81.

7. Dziewas R, Ritter M, Schilling M, Konrad C, Oelenberg S, Nabavi DG, et al. Pneumonia in acute stroke patients fed by nasogastric tube. J Neurol Neurosurg Psychiatry. 2004;75:852-6.

8. Montejo JC, Grau T, Acosta J, Ruiz-Santana S, Planas M, García-De-Lorenzo A, et al. Multicenter, prospective, randomized, single-blind study comparing the efficacy and gastrointestinal complications of early jejunal feeding with early gastric feeding in critically ill patients. Crit Care Med. 2002;30:796-800.

9. Wan B, Fu H, Yin J. Early jejunal feeding by bedside placement of a nasointestinal tube significantly improves nutritional status and reduces complications in critically ill patients versus enteral nutrition by a nasogastric tube. Asia Pac J Clin Nutr. 2015;24:51-7.

10. Strong RM, Condon SC, Solinger MR, Namihas BN, Ito-Wong LA, Leuty JE. Equal aspiration rates from postpylorus and intragastric-placed small-bore nasoenteric feeding tubes: A randomized, prospective study. J Parenter Enter Nutr. 1992. p. 59-63.

11. Kearns PJ, Chin D, Mueller L, Wallace K, Jensen WA, Kirsch CM. The incidence of ventilator-associated pneumonia and success in nutrient delivery with gastric versus small intestinal feeding: A randomized clinical trial. Crit Care Med. 2000;28:1742-6.

12. Davies AR, Morrison SS, Bailey MJ, Bellomo R, Cooper DJ, Doig GS, et al. A multicenter, randomized controlled trial comparing early nasojejunal with nasogastric nutrition in critical illness. Crit Care Med. 2012;40:2342-8.

13. Acosta-Escribano J, Fernández-Vivas M, Grau Carmona T, Caturla-Such J, Garcia-Martinez M, Menendez-Mainer A, et al. Gastric versus transpyloric feeding in severe traumatic brain injury: A prospective, randomized trial. Intensive Care Med. 
2010;36:1532-9.

14. Taylor SJ, Clemente R. Confirmation of nasogastric tube position by pH testing. J Hum Nutr Diet. 2005;18:371-5.

15. Alhazzani W, Almasoud A, Jaeschke R, Lo Y, Sindi BW, Altayyar A. S, et al. Small bowel feeding and risk of pneumonia in adult critically ill patients: A systematic review and meta-analysis of randomized trials. Crit Care. 2013;17:R127.

16. Deane Adam M, Rupinder D, Day Andrew G, Ridley Emma J, Davies Andrew R, Heyland Daren K. Comparisons between intragastric and small intestinal delivery of enteral nutrition in the critically ill: A systematic review and meta-analysis. Crit Care. 2013;17:R125.

17. Alberda C, Gramlich L, Jones N, Jeejeebhoy K, Day AG, Dhaliwal R, et al. The relationship between nutritional intake and clinical outcomes in critically ill patients: Results of an international multicenter observational study. Intensive Care Med. 2009;35:1728-37.

18. Hiesmayr M, Schindler K, Pernicka E, Schuh C, Schoeniger-Hekele A, Bauer P, et al. Decreased food intake is a risk factor for mortality in hospitalised patients: The NutritionDay survey 2006. Clin Nutr. 2009;28:484-91.

19. Allingstrup MJ, Esmailzadeh N, Wilkens Knudsen A, Espersen K, Hartvig Jensen T, Wiis J, et al. Provision of protein and energy in relation to measured requirements in intensive care patients. Clin Nutr. 2012;31:462-8.

20. Li Z, Qi J, Zhao X, Lin Y, Zhao S, Zhang Z, et al. Risk-benefit profile of gastric vs transpyloric feeding in mechanically ventilated patients. Nutr Clin Pract. 2016;31:91-8.

21. Lee JS, Jwa CS, Yi HJ, Chun YJ. Impact of early enteral nutrition on in-hospital mortality in patients with hypertensive intracerebral hemorrhage. J Korean Neurosurg Soc. 2010;48:99-104.

22. Rice TW, Mogan S, Hays MA, Bernard GR, Jensen GL, Wheeler AP. Randomized trial of initial trophic versus full-energy enteral nutrition in mechanically ventilated patients with acute respiratory failure. Crit Care Med. 2011. p. 967-74.

23. Nguyen NQ, Chapman MJ, Fraser RJ, Bryant LK, Burgstad C, Ching K, et al. The effects of sedation on gastric emptying and intra-gastric meal distribution in critical illness. Intensive Care Med. 2008;34:454-60.

24. Sun JK, Mu XW, Li WQ, Tong ZH, Li J, Zheng SY. Effects of early enteral nutrition on immune function of severe acute pancreatitis patients. World J Gastroenterol. 2013;19:917-22.

25. Mentec H, Dupont H, Bocchetti M, Cani P, Ponche F, Bleichner G. Upper digestive intolerance during enteral nutrition in critically ill patients: Frequency, risk factors, and complications. Crit Care Med. 2001;29:1955-61.

26. Balan KK, Vinjamuri S, Maltby P, Bennett J, Woods S, Playfer JR, et al. Gastroesophageal reflux in patients fed by percutaneous endoscopic gastrostomy (PEG): Detection by a simple scintigraphic method. Am J Gastroenterol. 1998;93:946-9.

27. Kompan L, Vidmar G, Spindler-Vesel A, Pečar J. Is early enteral nutrition a risk factor for gastric intolerance and pneumonia? Clin Nutr. 2004;23:527-32.

28. DeMeo MT, Bruninga K. Physiology of the Aerodigestive System and Aberrations in That System Resulting in Aspiration. J Parenter Enter Nutr. 2002;26:9-18.

29. Kuo B, Castell DO. The Effect of Nasogastric Intubation on Gastroesophageal Reflux: A Comparison of Different Tube Sizes. Am J Gastroenterol. 1995;90:1804-7.

30. Tryba M. The gastropulmonary route of infection - Fact or fiction? Am J Med. 1991. p. S135-46.

31. Jiyong J, Tiancha H, Huiqin W, Jingfen J. Effect of gastric versus post-pyloric feeding on the incidence of pneumonia in critically ill patients: Observations from traditional and Bayesian random-effects meta-analysis. Clin. Nutr. 2013. p. 8-15. 